\title{
In Vitro Genetic Transfer of Protein Synthesis and Respiration Defects to Mitochondrial DNA-Less Cells with Myopathy-Patient Mitochondria
}

\author{
ANNE CHOMYN,${ }^{1}$ GIOVANNI MEOLA ${ }^{2}$ NEREO BRESOLIN,${ }^{2}$ SUSAN T. LAI, ${ }^{1}$ \\ GUGLIELMO SCARLATO,$^{2}$ AND GIUSEPPE ATTARDI ${ }^{1 *}$ \\ Division of Biology, California Institute of Technology, Pasadena, California 91125, ${ }^{1}$ \\ and Institute of Clinical Neurology, University of Milan, 20122 Milan, Italy
}

Received 8 November 1990/Accepted 11 January 1991

\begin{abstract}
A severe mitochondrial protein synthesis defect in myoblasts from a patient with mitochondrial myopathy was transferred with myoblast mitochondria into two genetically unrelated mitochondrial DNA (mtDNA)-less human cell lines, pointing to an mtDNA alteration as being responsible and sufficient for causing the disease. The transfer of the defect correlated with marked deficiencies in respiration and cytochrome $c$ oxidase activity of the transformants and the presence in their mitochondria of mtDNA carrying a tRNA ${ }^{\text {Lys }}$ mutation. Furthermore, apparently complete segregation of the defective genotype and phenotype was observed in the transformants derived from the heterogeneous proband myoblast population, suggesting that the mtDNA heteroplasmy in this population was to a large extent intercellular. The present work thus establishes a direct link between mtDNA alteration and a biochemical defect.
\end{abstract}

The genetic analysis of mitochondrial diseases has been complicated by the existence of dual control by nuclear and mitochondrial DNA (mtDNA) of mitochondrion formation and by the interplay of the two genomes in this process (3). Another difficulty in the study of the diseases associated with mtDNA mutations has been the variable degree of penetrance of the defect $(25,27,32)$. This variability reflects the frequent coexistence in the same individual of mutated and nonmutated mtDNA in varying proportions, often with tissue-to-tissue differences $(11-13,23,27,29,34)$, as well as the influence of the nuclear background in the phenotypic manifestation of the mtDNA-encoded defect $(15,27,32)$. Also, the molecular dissection of the defect has been hindered by the pleiomorphic character of the clinical syndromes and the striking tissue specificity of the mitochondrial dysfunction $(5,8,20,31)$.

We have recently developed novel approaches for mitochondrion-mediated transformation of human cells which can find application in the genetic and molecular analysis of mitochondrial diseases. In particular, several human cell lines completely devoid of mtDNA $\left(\rho^{\circ}\right)$ have been isolated by long-term exposure of the cells to low concentrations of ethidium bromide (15). Owing to the lack of a functional respiratory chain, these cell lines have become auxotrophic for pyrimidines and pyruvate. Loss of either of these two requirements has been used as a selectable marker for the repopulation of the human $\rho^{\circ}$ cell lines with exogenous mitochondria from different human sources (15).

In the present study, the methodology described above was utilized to investigate the genetic and molecular basis of a mitochondrial protein synthesis defect found in myoblasts from a patient affected by a mitochondrial myopathy. The results clearly show (i) that the genetic defect responsible for the protein synthesis alteration could be transferred to different human mtDNA-less cell lines with the patient's mitochondria, and therefore resided in mtDNA; (ii) that the

\footnotetext{
* Corresponding author.
}

transformants obtained with different myoblasts from the proband contained either mutated or wild-type mtDNA, pointing to mtDNA heteroplasmy in the myoblasts which was probably to great extent intercellular; and (iii) that the occurrence of the protein synthesis alteration in the transformants was correlated with a marked respiratory deficiency. Furthermore, DNA sequence analysis revealed the presence of the tRNA ${ }^{\text {Lys }}$ mutation, recently found in patients suffering from myoclonic epilepsy and ragged red fiber (MERRF) syndrome (27), in the mtDNA of the transformants derived from the defective myoblasts of the proband.

\section{MATERIALS AND METHODS}

Cell lines and media. The $\rho^{\circ} 206$ cell line (15), a derivative of $143 \mathrm{~B} . \mathrm{TK}^{-}$cells, was grown in Dulbecco modified Eagle medium (DMEM) with $4.5 \mathrm{mg}$ of glucose per $\mathrm{ml}$, supplemented with $5 \%$ fetal bovine serum (FBS), $50 \mu \mathrm{g}$ of uridine per $\mathrm{ml}$, and $100 \mu \mathrm{g}$ of 5-bromodeoxyuridine (BrdU) per $\mathrm{ml}$. The $\rho^{\circ} 701.2$ a cell line was obtained from the simian virus 40 T-antigen gene-transformed derivative (701.2.8c [33a]) of the human fibroblast strain GM701 (10) by long-term exposure to a low concentration of ethidium bromide (14a). It was grown in DMEM with $10 \% \mathrm{FBS}$ and $50 \mu \mathrm{g}$ of uridine per $\mathrm{ml}$. The $\rho^{\circ} 13.1$ cell line was obtained by long-term exposure to ethidium bromide of an adenine phosphoribosyltransferaseless mutant of 143B. $\mathrm{TK}^{-}$(isolated by two-step selection in the presence of 8-azaadenine [1a]). It was grown in DMEM with $10 \%$ FBS, $50 \mu \mathrm{g}$ of uridine per $\mathrm{ml}$, and $50 \mu \mathrm{g}$ of 8 -azaadenine per ml. 143B. $\mathrm{TK}^{-}$cells were grown as previously described (15).

Myoblast cultures and isolation of myoblast clones. Mass myoblast cultures were established by a previously established procedure (16), using muscle biopsy specimens from the proband and from four normal adult individuals. Myoblast clones were isolated as previously reported (17) by plating a mass myoblast culture at a low density ( $\sim 200$ cells per $10-\mathrm{cm}$ dish) in conditioned medium, identifying the 


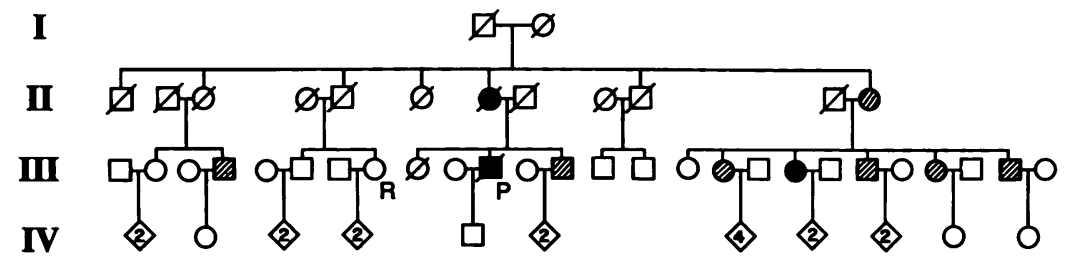

FIG. 1. Pedigree of the family of the proband analyzed in this work. Symbols: $\bullet$, MERRF syndrome; $\square$, mitochondrial myopathy; $\bullet$, , ragged red fibers and/or other laboratory abnormalities; $\bigcirc, \square$, apparent absence of clinical manifestations; Diagonal lines indicate deaths. $\mathbf{P}$, Proband; R, relative analyzed in the present work. See text for details.

myogenic colonies by the appearance of initial fusion, and trypsinizing them in a glass ring.

Mitochondrion-mediated transformation. $\rho^{\circ}$ cell transformation by cytoplast fusion was done as previously described (15). Approximately 6,000 proband myoblasts from a mixed culture of five clones were enucleated (microscopic inspection of the cytoplast preparation revealed the great efficiency of the enucleation step [ $>90 \%$ ]) and fused to $\sim 2 \times 10^{6} \rho^{\circ} 206$ cells, and transformants were isolated in DMEM supplemented with $5 \%$ dialyzed FBS and $100 \mu \mathrm{g}$ of BrdU per ml. In other experiments, two such transformants were fused to $\rho^{\circ} 13.1$ cells as detailed in the text, using DMEM supplemented with $10 \%$ dialyzed FBS and $50 \mu \mathrm{g}$ of 8 -azaadenine per $\mathrm{ml}$ as a selective medium. In still other experiments, cytoplasts from several hundred to several thousand cells of each of five myoblast clones from the proband (1 to 5) were fused to a 200 - to 2,000 -fold excess of $\rho^{\circ} 206$ cells or $\rho^{\circ} 701.2 \mathrm{a}$ cells, and transformants were isolated in DMEM with 5\% dialyzed FBS.

Mitochondrial protein synthesis analysis. Samples of mass myoblast cultures from the proband or control individuals, of a mixed culture of five myoblast clones, or of individual transformants were labeled with $\left[{ }^{35} \mathrm{~S}\right]$ methionine $(1,000 \mathrm{Ci} /$ mmol, 125 to $500 \mu \mathrm{Ci} / \mathrm{ml}$ ) for 1.2 to $2 \mathrm{~h}$, as detailed in the figure legends, in the presence of $100 \mu \mathrm{g}$ of emetine per $\mathrm{ml}$ in methionine-free DMEM supplemented with $10 \%$ dialyzed FBS. 143B cells were similarly labeled for $1.2 \mathrm{~h}$. After the labeling, 2 volumes of complete DMEM were added, and the cells were then washed once with DMEM and twice with buffered saline lacking $\mathrm{Mg}^{2+}$ and $\mathrm{Ca}^{2+}$ (TD), trypsinized, washed twice with TD, and finally resuspended in TD containing $1 \mathrm{mM}$ phenylmethylsulfonyl fluoride and lysed with $1 \%$ sodium dodecyl sulfate (SDS). In some experiments, cells were labeled in 16-mm microwells, washed, and directly lysed in $10 \mathrm{mM}$ Tris ( $\mathrm{pH}$ 7.3)-0.1 mM EDTA-1 mM phenylmethylsulfonyl fluoride containing 1\% SDS. Samples of the various cell suspensions were run on SDS-exponential polyacrylamide gradient gels (7). In some experiments, a sample of an SDS lysate of the mitochondrial fraction from 2-h $\left[{ }^{35} \mathrm{~S}\right]$ methionine-labeled HeLa cells, prepared as previously described (6), was run in parallel.

DNA analysis. Total DNA was prepared from cells by using an Applied Biosystems 340A DNA extractor. Equal samples were digested with restriction endonucleases as recommended by the suppliers, electrophoresed in a $0.7 \%$ agarose gel ( $\mathrm{XbaI}$ or $\mathrm{NcoI}$ digests) or in a $5 \%$ polyacrylamide gel ( $M b o I$ digest), and transferred by capillarity or electroblotted onto a Nytran nylon membrane $(0.2-\mu \mathrm{m}$ pore size; Schleicher \& Schuell). The XbaI and NcoI blots were probed with total HeLa cell mtDNA, ${ }^{32} \mathrm{P}$ labeled by random priming (9). The MboI blot was probed with the mtDNA clone mp18.XK76, containing the 1,097-bp KpnI-XhoI fragment of HeLa mtDNA between positions 14956 and 16053 of the Cambridge sequence (1) inserted in the KpnI and SalI sites of M13mp18 (14a), ${ }^{32} \mathrm{P}$ labeled by random priming. For the polymerase chain reaction, samples of total cell DNA (10 to $300 \mathrm{ng}$ ) were processed in a thermal cycler with Taq polymerase (Amersham) and oligonucleotides corresponding to the light strand between positions 8100 and 8117 and to the heavy strand between positions 8392 and 8372 as primers. A light-strand primer corresponding to positions 8263 to 8283 was used for sequencing by the dideoxynucleotide-chain termination method (26).

$\mathrm{O}_{2}$ consumption and $\mathrm{COX}$ measurements. $\mathrm{O}_{2}$ consumption was measured with a Gilson 5/6 oxygraph on two to four samples of $2 \times 10^{6}$ to $5 \times 10^{6}$ cells in $1.85 \mathrm{ml}$ of DMEM lacking glucose, supplemented with $5 \%$ dialyzed calf serum. Cytochrome $c$ oxidase (COX) activity was measured in duplicate to quadruplicate on two to three samples of isolated mitochondrial fraction as previously described (28). The mtDNA concentration of these samples was determined by dot-blot analysis as described elsewhere (15).

Chromosome analysis. Cells were arrested in metaphase by treatment with $0.05 \mu \mathrm{g}$ of colchicine per $\mathrm{ml}$ for 3 to $5 \mathrm{~h}$ or with $0.1 \mu \mathrm{g}$ of vinblastine sulfate per ml for 15 to $22 \mathrm{~h}$. Karyotype analysis was done as described previously (18).

\section{RESULTS}

Protein synthesis defect in proband myoblasts. Figure 1 shows the pedigree of the family to which the proband $(P)$ belonged (4). In this family, two members exhibited a typical MERRF syndrome, with myoclonus and episodes of generalized tonic-clonic seizures, progressive myopathy with ragged red fibers in the muscle biopsy specimen, deafness, and cerebellar abnormalities. Other members, including the proband, exhibited mainly myopathy symptoms, ragged red fibers, lactic acidosis, and electromyogram abnormalities. Other members had no obvious clinical symptoms but some laboratory signs of disease, such as ragged red fibers, lactic acidosis, and electrocardiogram or electroencephalogram abnormalities. Finally, other members appeared normal at the clinical and laboratory examination. All affected members were related through maternal lineages. The clinical and biochemical characterization of the pedigree will be reported elsewhere (4a).

A histochemical analysis of a muscle biopsy specimen from the proband had previously revealed the presence of ragged red fibers (about $40 \%$ of the fibers) and COX-negative and succinate dehydrogenase-positive fibers (about $30 \%$ of the fibers) (4a). Furthermore, a Southern blot analysis of the muscle DNA had failed to show the presence of any gross deletions of mtDNA. In the present work, a mass myoblast culture from the proband was tested for mitochondrial protein synthesis, in parallel with mass myoblast cultures from three normal individuals. The pattern of the mitochon- 


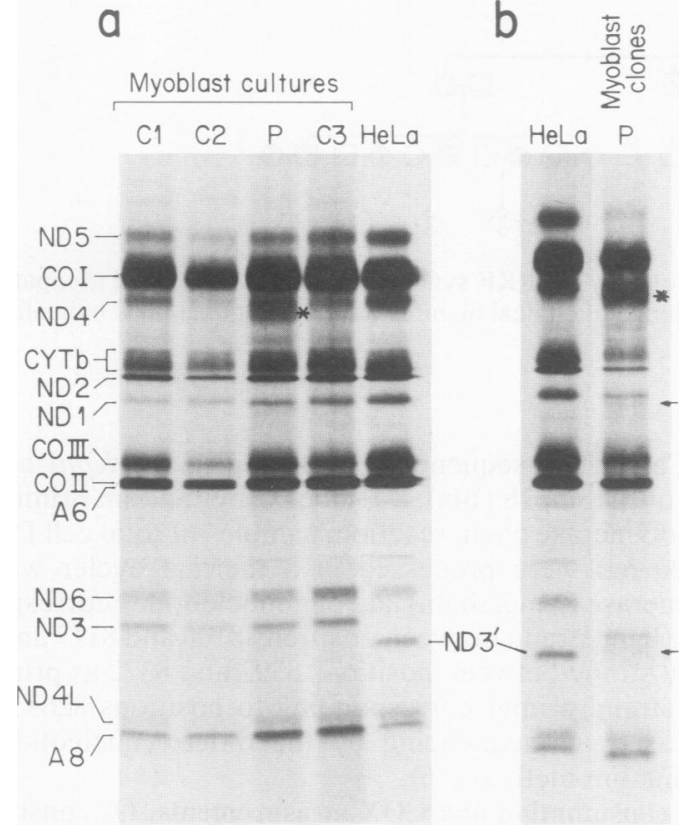

FIG. 2. Myoblast cultures from the proband exhibit an abnormal pattern of mitochondrial protein synthesis. Shown are fluorograms, after electrophoresis through SDS-polyacrylamide gradient gels, of the mitochondrial translation products labeled with $\left[{ }^{35} \mathrm{~S}\right]$ methionine in mass myoblast cultures from the proband $(P)$ and three different normal individuals $(\mathrm{C} 1, \mathrm{C} 2$, and $\mathrm{C} 3)$ and in HeLa cells (a) and in a culture of mixed myoblast clones from the proband $(\mathrm{P})$ and in HeLa cells (b). The cultures were labeled with $\left.{ }^{35} \mathrm{~S}\right]$ methionine in the presence of emetine (for $2 \mathrm{~h}$ in panel a and $1.6 \mathrm{~h}$ in panel b), and samples of total cell lysates of myoblast cultures $(15 \mu \mathrm{g}$ of protein in panel a and $48 \mu \mathrm{g}$ in panel b) or of a lysate of the mitochondrial fraction (HeLa cells) were run through SDS-polyacrylamide gradient gels. COI, COII, and COIII: subunits I, II, and III of COX; ND1, ND2, ND3, ND4, ND4L, ND5, and ND6, subunits 1, 2, 3, 4, $4 \mathrm{~L}, 5$, and 6 of the respiratory chain NADH dehydrogenase; $\mathrm{A} 6$ and A8, subunits 6 and 8 of the $\mathrm{H}^{+}$-ATPase; CYTb, apocytochrome B.

drial translation products from the proband was indistinguishable from that of the three control myoblast cultures, except for the presence of a band migrating slightly faster than ND4 (Fig. 2a, asterisk). Figure $2 \mathrm{a}$ also shows the mitochondrial translation pattern from HeLa cells, which differs from that of other human cells for a previously described ND3 polymorphism (22). That the abnormal pattern of mitochondrial protein synthesis observed in the mass myoblast culture from the proband was due to the predominant myoblasts in the culture and not to fibroblasts was shown by the experiment illustrated in Fig. 2b. In this experiment, the mitochondrial translation products of a mixture of five myoblast clones isolated from the mass myoblast culture were analyzed in an SDS-polyacrylamide gel, in parallel with the mitochondrial translation products of HeLa cells. The pattern from the proband myoblast clones again exhibited the abnormal band migrating faster than ND4, as well as two minor abnormal bands (indicated by small arrows).

Phenotype transfer with proband mitochondria. To investigate whether the abnormal pattern of mitochondrial protein synthesis observed in the proband myoblasts was due to an mtDNA or a nuclear DNA mutation, we fused enucleated cells (cytoplasts) from the proband myoblast clone mixture, in the presence of polyethylene glycol, with a large excess of the $\rho^{\circ} 206$ derivative of the BrdU-resistant 143 B. TK $^{-}$cells (15). Many colonies of presumptive transformants were observed after 10 days of growth of the cell fusion mixture in medium containing BrdU and lacking uridine. In this medium, only fusion products of myoblast cytoplasts and $\rho^{\circ} 206$ cells were expected to survive, since $\rho^{\circ} 206$ cells would not grow in the absence of uridine and, on the other hand, any residual nonenucleated myoblasts or hybrids between these and $\rho^{\circ} 206$ cells would be killed by BrdU, converted to its toxic phosphorylated derivative by the thymidine kinase encoded in the myoblast nucleus. A karyotype analysis of two transformants (pT1, pT3) revealed ranges of chromosome numbers $(72$ to 93,76 to 86$)$ very similar to that of $\rho^{\circ} 206$ cells (70 to 97), confirming their cybrid nature.

Five cybrid clones (pT1 to pT5) were tested for mitochondrial protein synthesis. Two of the clones, pT1 and pT4, exhibited a pattern of mitochondrial translation products with the same abnormalities observed in the proband myoblasts, whereas the three remaining transformants exhibited a normal pattern (Fig. 3a). A transformant obtained by fusion of $\rho^{\circ} 206$ cells with cytoplasts from a normal individual (C4) also showed a normal protein synthesis pattern. These findings provided the first evidence indicating that the protein synthesis defect was due to an mtDNA mutation. A plausible interpretation of the results was that the mtDNA mutation was heteroplasmic and that this heteroplasmy was to great extent intercellular, some myoblasts containing exclusively or mainly defective mtDNA, and others containing exclusively or mainly normal mtDNA. However, selection phenomena during the growth of the myoblasts and of the transformants could have played a role in the final genotype and phenotype of the transformants. On the other hand, the data did not exclude the possibility that the phenotypic heterogeneity of the transformants was due to nuclear heterogeneity of the $\rho^{\circ} 206$ recipient cells. To test the latter possibility, we did experiments in which one defective transformant, pT1, and one apparently normal transformant, pT3, were used as mitochondrial donors in a second cycle of transformation experiments. In these experiments, another $\rho^{\circ}$ cell line, $\rho^{\circ} 13.1$, derived as described elsewhere (14a) from an adenine phosphoribosyltransferase-less mutant of 143B. $\mathrm{TK}^{-}$by long-term exposure to a low concentration of ethidium bromide, was used as a recipient. The 8-azaadenine resistance of this cell line provided a convenient selectable marker to discriminate between the mitochondrial transformants and any residual nonenucleated $\rho^{\circ} 206$ transformants or hybrids of these with $\rho^{\circ} 13.1$, since the latter were expected to be sensitive to the drug. In two fusion experiments with $\sim 1.5 \times 10^{5}$ pT1 or pT3 cells and a 10-fold excess of $\rho^{\circ} 13.1$ cells, 10 or 9 transformant colonies, respectively, were isolated in selective medium represented by DMEM supplemented with $50 \mu \mathrm{g}$ of 8-azaadenine per ml. In a control experiment with $3.1 \times 10^{6}$ cells of a $\rho^{\circ} 206$ transformant derived from a proband myoblast clone (no. 2, see below), no 8-azaadenine-resistant spontaneous mutants appeared during a 3-week culture in the presence of $50 \mu \mathrm{g}$ of the drug per ml. This experiment strongly argued against the possibility of the putative $\rho^{\circ} 13.1$ transformants being instead spontaneous 8-azaadenine-resistant mutants of the $\rho^{\circ} 206$ transformants. Five $\rho^{\circ} 13.1$ transformants obtained with pT1, when tested for mitochondrial protein synthesis, revealed a defective pattern (Fig. 4), with the characteristic abnormal bands first observed in the myoblasts. Similarly, five $\rho^{\circ} 13.1$ transformants obtained with pT3 showed a normal pattern of mitochondrial translation products. These experiments thus clearly showed that the basis for the phenotypic heteroge- 
a

b

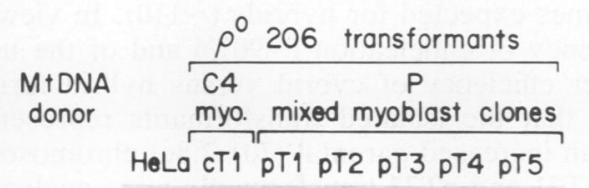

\section{Recipient \\ MtDNA \\ donor \\ donor}
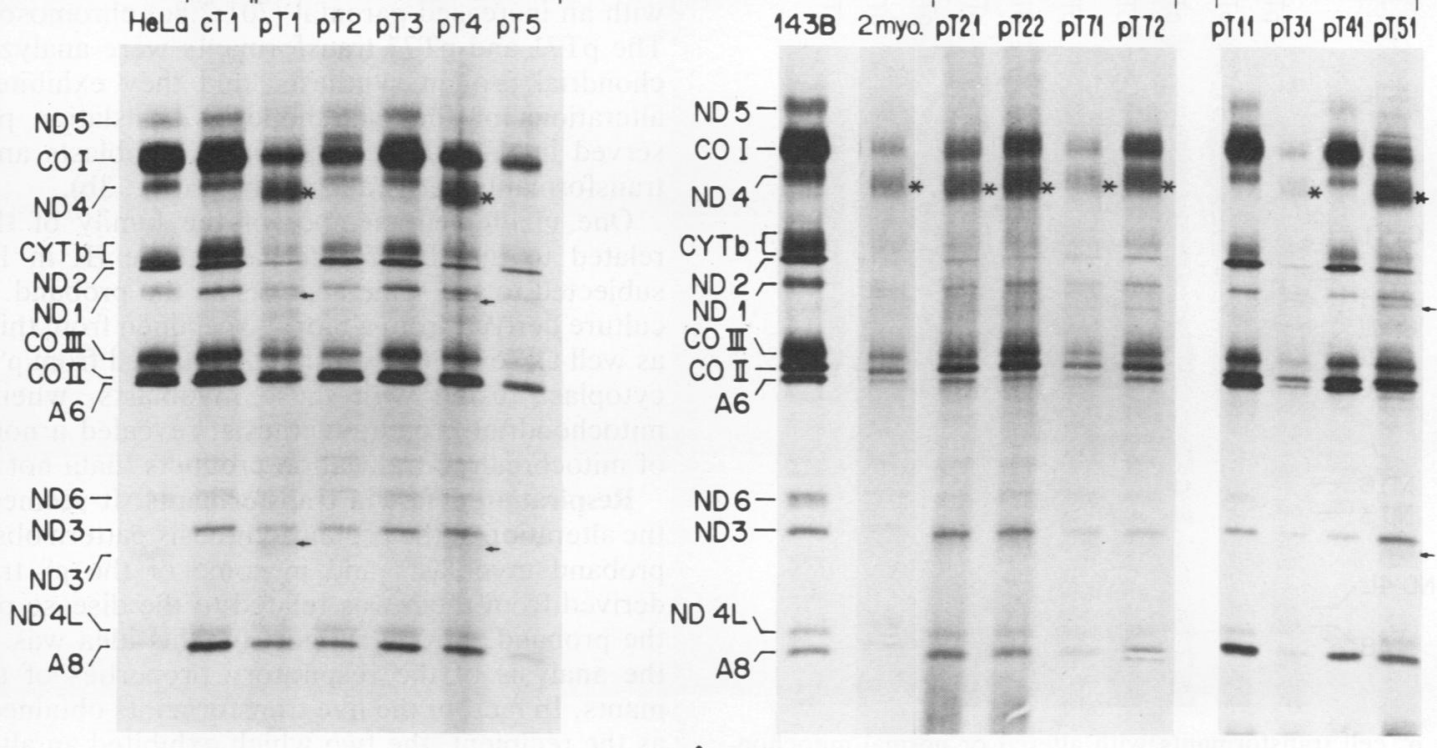

C
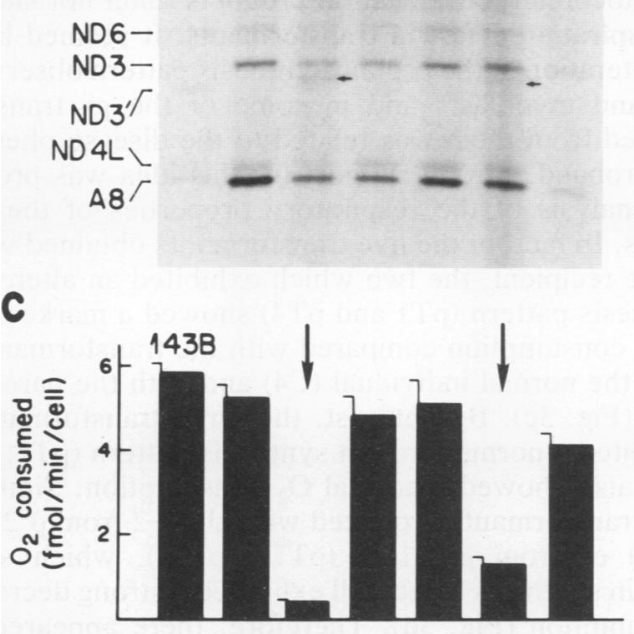

d
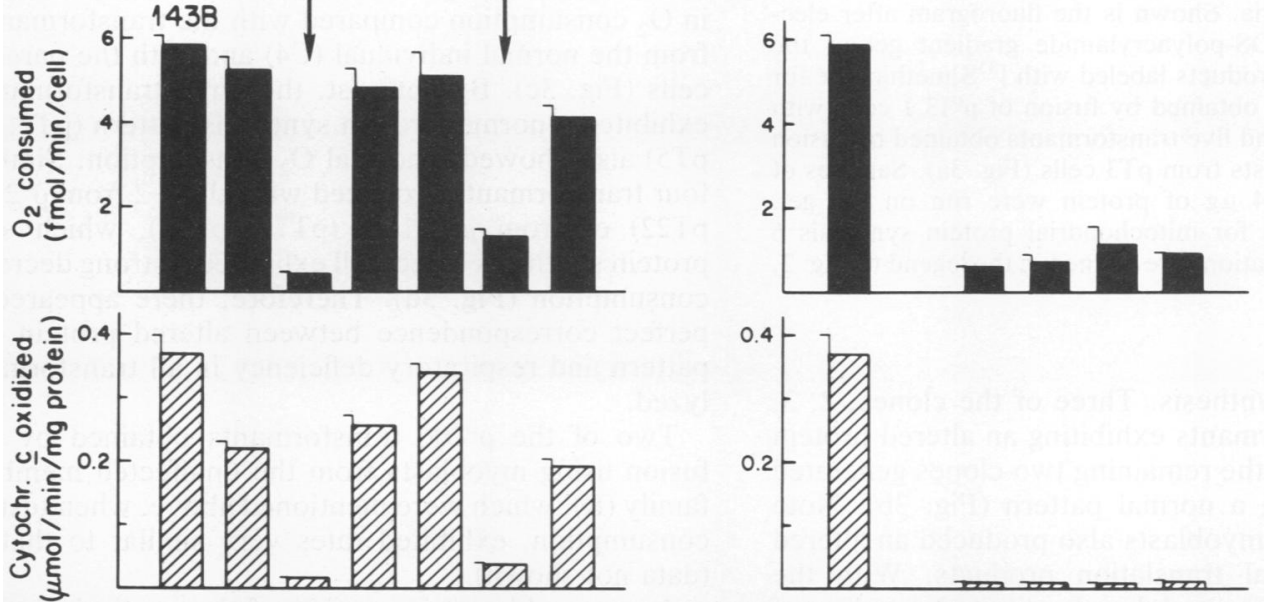

FIG. 3. Transformants obtained by mitochondrion transfer from proband myoblasts into human $\rho^{\circ}$ cells are either defective in mitochondrial protein synthesis and respiratory capacity or have a normal phenotype. Panel a shows the pattern of mitochondrial translation products $\left(1.3 \mathrm{~h}\right.$ of labeling, $30 \mu \mathrm{g}$ of protein run on gel) and panel c shows the rate of $\mathrm{O}_{2}$ consumption and the level of COX activity (with error bars representing two standard errors) in five mitochondrial transformants (pT1 to pT5) generated from $\rho^{\circ} 206$ cells by fusion with cytoplasts from a mixture of five myoblast clones from the proband (Fig. 2), in one $\rho^{\circ} 206$ transformant (cT1) obtained with myoblasts from a normal individual (C4), and, for comparison, in HeLa cells and 143B cells. Panel b shows the protein synthesis pattern $(1.3 \mathrm{~h} \mathrm{of} \mathrm{labeling,} 10 \mu \mathrm{g}$ of protein run on gel) and panel d shows the rate of $\mathrm{O}_{2}$ consumption and the level of COX activity in $\rho^{\circ} 206$ transformants obtained with five additional individual myoblast clones from the proband (clones 1 to 5), in two $\rho^{\circ} 701.2 \mathrm{a}$ transformants obtained with myoblast clone 2, and, for comparison, in 143B cells. Transformants were tested for mitochondrial protein synthesis, $\mathrm{O}_{2}$ consumption, and COX activity 4 to 6 weeks, 8 to 12 weeks, and 8 to 14 weeks, respectively, after fusion. Abbreviations are defined in the legend to Fig. 2.

neity of the transformants resided in the mtDNA heteroplasmy of the proband myoblasts.

The experiments described above, in which two of five transformants analyzed revealed an altered protein synthesis pattern, did not provide precise information concerning the proportion of myoblasts with exclusively or predominantly defective mtDNA in the myoblast clone mixture tested. An estimate of the frequency of the defective myoblasts was provided by another experiment in which five additional myoblast clones, isolated independently from the original myoblast culture, were analyzed individually. After cytoplasts from each one of clones 1 to 5 were fused to a large excess of $\rho^{\circ} 206$ cells, several transformants were isolated. Karyotype analysis of four transformants revealed a range of chromosome numbers (69 to 90 ) very similar to that of $\rho^{\circ} 206$ (70 to 97), as expected for cybrids. The original clone 2 and two transformants produced with it and one transformant obtained with each one of the other clones were analyzed for 


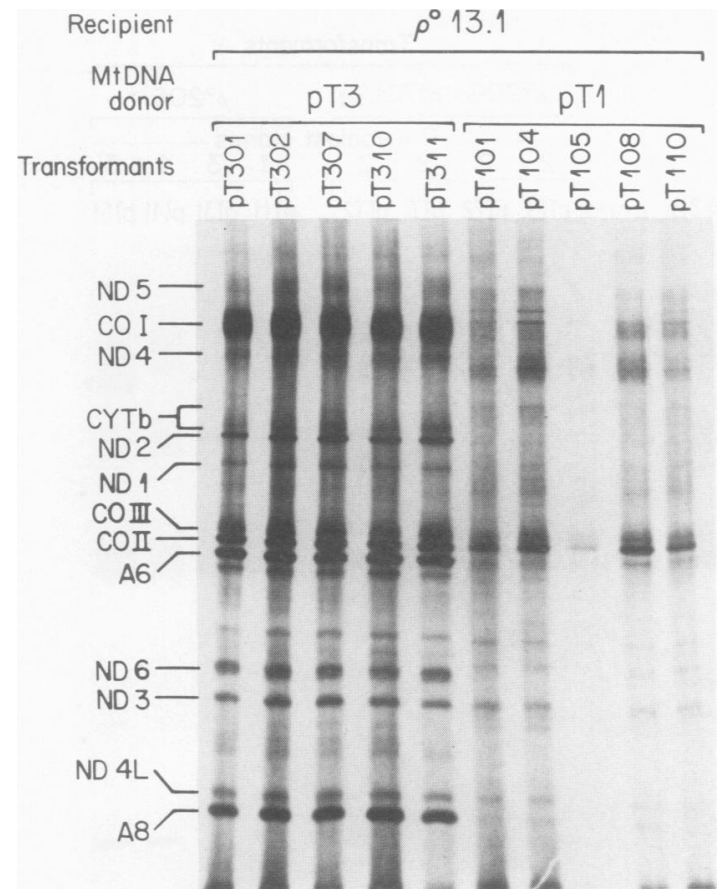

FIG. 4. $\rho^{\circ}$ cell transformants with altered or normal mitochondrial protein synthesis pattern transfer their phenotype to $\rho^{\circ} 13.1$ cells with their mitochondria. Shown is the fluorogram after electrophoresis through an SDS-polyacrylamide gradient gel of the mitochondrial translation products labeled with $\left[{ }^{35} \mathrm{~S}\right]$ methionine for $1.3 \mathrm{~h}$ in five transformants obtained by fusion of $\rho^{\circ} 13.1$ cells with cytoplasts from pT1 cells and five transformants obtained by fusion of $\rho^{\circ} 13.1$ cells with cytoplasts from pT3 cells (Fig. 3a). Samples of SDS lysates containing $3.4 \mu \mathrm{g}$ of protein were run on the gel. Transformants were tested for mitochondrial protein synthesis 6 weeks after fusion. Abbreviations are defined in the legend to Fig. 2.

mitochondrial protein synthesis. Three of the clones, 2, 3, and 5 , produced transformants exhibiting an altered protein synthesis pattern, while the remaining two clones generated transformants exhibiting a normal pattern (Fig. 3b). Note that the original clone 2 myoblasts also produced an altered pattern of mitochondrial translation products. With the obvious qualifications imposed by the limited number of clones analyzed, this experiment, together with the previous one, thus suggested that about $50 \%$ of the myoblast clones were derived from myoblasts with exclusively or predominantly defective mtDNA. This may be an underestimate of the proportion of the defective myoblasts in the original muscle culture, considering the probable selective advantage for clonal growth and for transforming capacity of the myoblasts with predominantly wild-type mtDNA. Myoblast clone 2 was also used to transform another $\rho^{\circ}$ human cell line, $\rho^{\circ} 701.2 \mathrm{a}$, genetically unrelated to $143 \mathrm{~B} . \mathrm{TK}^{-}$, which exhibited the uridine and pyruvate dependence previously shown for $\rho^{\circ} 206$ and $\rho^{\circ} 101(2,15)$. Several transformants were obtained from $\rho^{\circ} 701.2 \mathrm{a}$ by fusion with cytoplasts from clone 2 and selection in medium not supplemented with uridine. In this medium, only cybrids between clone 2 myoblasts and $\rho^{\circ} 701.2 \mathrm{a}$ cells and possibly hybrids involving any residual nonenucleated myoblasts were expected to survive. Karyotype analysis of two transformants (pT71, pT72) revealed chromosome numbers (69 to 108,85 to 109) higher than the range of values observed in $\rho^{\circ} 701.2 \mathrm{a}$ cells $(60$ to 68), although in general lower than the number of chromosomes expected for hybrids $(\sim 110)$. In view of the high frequency of enucleation $(>90 \%)$ and of the usually much greater efficiency of cybrid versus hybrid formation, it is likely that the isolated transformants represented cybrids with an increased parental (701.2.8c) chromosome number. The pT71 and pT72 transformants were analyzed for mitochondrial protein synthesis, and they exhibited the same alterations of the mitochondrial translation products observed in the defective proband myoblasts and the $\rho^{\circ} 206$ transformants derived from them (Fig. 3b).

One unaffected member of the family of the proband, related to him by a paternal lineage ( $R$ in Fig. 1), was subjected to the same analysis as the proband. A myoblast culture derived from a biopsy specimen from this individual, as well as seven transformants obtained from $\rho^{\circ} 206$ cells by cytoplast fusion with these myoblasts, when tested for mitochondrial protein synthesis, revealed a normal pattern of mitochondrial translation products (data not shown).

Respiration defect in transformants. It seemed likely that the alteration in the protein synthesis pattern observed in the proband myoblasts and in some of the $\rho^{\circ}$ transformants derived from them was related to the disease phenotype of the proband. Strong support for this idea was provided by the analysis of the respiratory properties of the transformants. In fact, of the five transformants obtained with $\rho^{\circ} 206$ as the recipient, the two which exhibited an altered protein synthesis pattern (pT1 and pT4) showed a marked decrease in $\mathrm{O}_{2}$ consumption compared with the transformant derived from the normal individual (C4) and with the parental 143B cells (Fig. 3c). By contrast, the three transformants which exhibited a normal protein synthesis pattern (pT2, pT3, and pT5) also showed a normal $\mathrm{O}_{2}$ consumption. Similarly, the four transformants produced with clone 2 from $\rho^{\circ} 206$ (pT21, pT22) or from $\rho^{\circ 701.2 a ~(p T 71, ~ p T 72), ~ w h i c h ~ s h o w e d ~ a ~}$ protein synthesis defect, all exhibited a strong decrease in $\mathrm{O}_{2}$ consumption (Fig. 3d). Therefore, there appeared to be a perfect correspondence between altered protein synthesis pattern and respiratory deficiency in all transformants analyzed.

Two of the $\rho^{\circ} 206$ transformants obtained by cytoplast fusion using myoblasts from the unaffected member of the family (R), which were mentioned above, when tested for $\mathrm{O}_{2}$ consumption, exhibited rates very similar to that of $143 \mathrm{~B}$ (data not shown).

A reasonable interpretation of the respiratory deficiency observed in the defective transformants was that it was due to the marked decrease, as a result of the severe protein synthesis defect, in the rate of assembly of functional respiratory complexes which are under dual (mitochondrial and nuclear) genetic control. This interpretation was supported by the results of measurements of activity of one such complex, COX, in the transformants. All transformants exhibiting a strong reduction in the rate of $\mathrm{O}_{2}$ consumption also showed a pronounced decrease in COX activity per milligram of mitochondrial protein, relative to the parental 143B cells (Fig. 3c and d). Similarly, all transformants with normal $\mathrm{O}_{2}$ consumption exhibited a normal COX activity. Normalization of the COX data for the mtDNA content of the mitochondrial fraction, determined by dot-blot analysis as previously described (15), yielded relative values of COX activity in the $\rho^{\circ} 206$ transformants and 143B cells very similar to the values normalized per protein content (data not shown).

Nature of mtDNA defect in proband myoblasts. The analysis of the mitochondrial translation products in the proband 


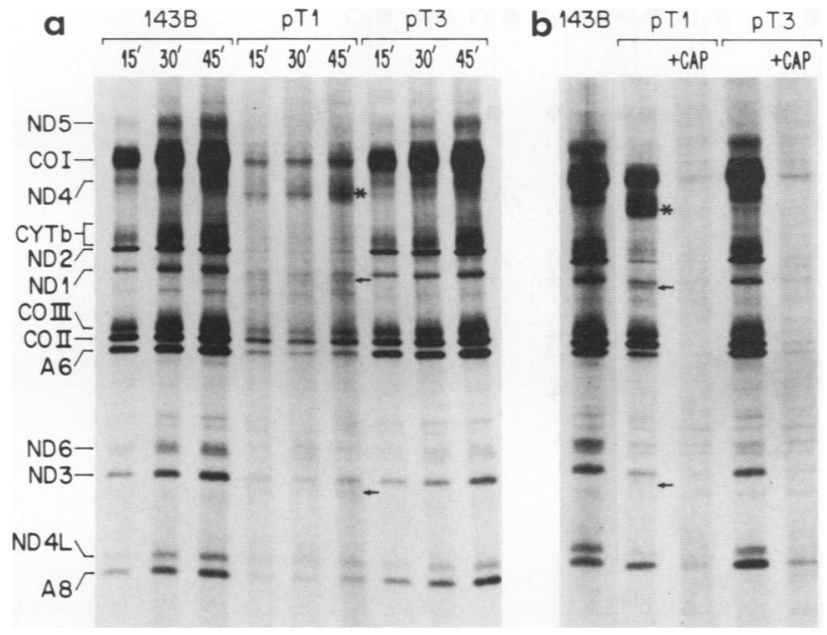

FIG. 5. The $\rho^{\circ}$ cell transformants with an altered pattern of mitochondrial protein synthesis exhibit a markedly decreased rate of CAP-sensitive translation. Panel a shows the pattern of mitochondrial translation products of the transformants pT1 and pT3 and of $143 B$, labeled with $\left[{ }^{35} S\right]$ methionine for different times. Samples of the SDS lysates containing equal amounts of protein $(20 \mu \mathrm{g})$ were run on an SDS-polyacrylamide gradient gel. Panel b shows the effects of CAP $(100 \mu \mathrm{g} / \mathrm{ml})$ on the labeling of the mitochondrial translation products of $\mathrm{pT} 1$ and $\mathrm{pT} 3$ during a 1.2-h exposure of the cells to ${ }^{35} \mathrm{~S}$ ]methionine in the presence of $100 \mu \mathrm{g}$ of emetine per $\mathrm{ml}$. Samples containing $50 \mu \mathrm{g}$ of protein were run on a gel in parallel with a sample of total cell lysate of $\left[{ }^{35}\right.$ S $]$ methionine-labeled 143B cells. Abbreviations are defined in the legend to Fig. 2.

myoblasts and in the transformants derived therefrom revealed the presence of several abnormal bands. This suggested that the mtDNA defect did not involve any single protein-coding gene. To investigate whether the abnormal translation products derived from degradation phenomena, we did an experiment in which pT1, pT3, and 143B cells were subjected to short $\left[{ }^{35} \mathrm{~S}\right]$ methionine labeling pulses.
Surprisingly, mitochondrial protein synthesis was found to be severely decreased in pT1 compared with $143 \mathrm{~B}$ and pT3 (Fig. 5a). In particular, the labeling of the various mitochondrial translation products in pT1 after a 30 -min pulse, as determined by densitometric analysis of the autoradiogram, was 2 to $20 \%$ of that in $143 \mathrm{~B}^{-\mathrm{TK}^{-}}$and $<4$ to $36 \%$ of that in pT3. The higher-molecular-weight products tended to be more affected than those of lower molecular weight. Furthermore, the abnormal translation products were labeled in the same proportion, relative to the normal products, after a short pulse as after a 1.3- or 2-h pulse, arguing against their derivation from degradation phenomena. The above observations pointed to a defect of the translation apparatus, i.e., either a ribosome defect or a tRNA alteration. In previous work (33), mtDNA-encoded mutants of the human $\mathrm{VA}_{2}-\mathrm{B}$ cells which were defective in mitochondrial protein synthesis due to an alteration(s) in mitochondrial ribosome assembly had been found to exhibit a substantial resistance to chloramphenicol (CAP) of the residual protein synthesis. Therefore, we examined the CAP sensitivity of mitochondrial translation in $\mathrm{pT} 1$. The residual protein synthesis of pT1 was found to be as sensitive to CAP inhibition as the protein synthesis of $\mathrm{pT} 3$ (Fig. 5b). In another experiment, in which the rate of $\left[{ }^{3} \mathrm{H}\right]$ uridine incorporation into the mitochondrial ribosomal subunits $45 \mathrm{~S}$ and $35 \mathrm{~S}$, separated by sucrose gradient centrifugation (33), was directly analyzed, the assembly of these subunits in pT1 was found not to be affected when compared to the assembly in pT3 or 143B (data not shown). The above observations tended to exclude a ribosomal involvement in the pT1 defect and pointed to a tRNA gene as the probable site of the mutation.

A Southern blot analysis of total cell DNA from pT1, pT3, and $143 \mathrm{~B}$, digested with $\mathrm{XbaI}$ and probed with HeLa cell mtDNA ${ }^{32} \mathrm{P}$ labeled by random priming, revealed all the expected fragments in the same relative amounts in the three samples (Fig. 6a), thus showing the absence of any major mtDNA deletion in the transformants, in confirmation of what had been previously found in the proband muscle biopsy specimen. Similar results were obtained with $\mathrm{NcoI}$

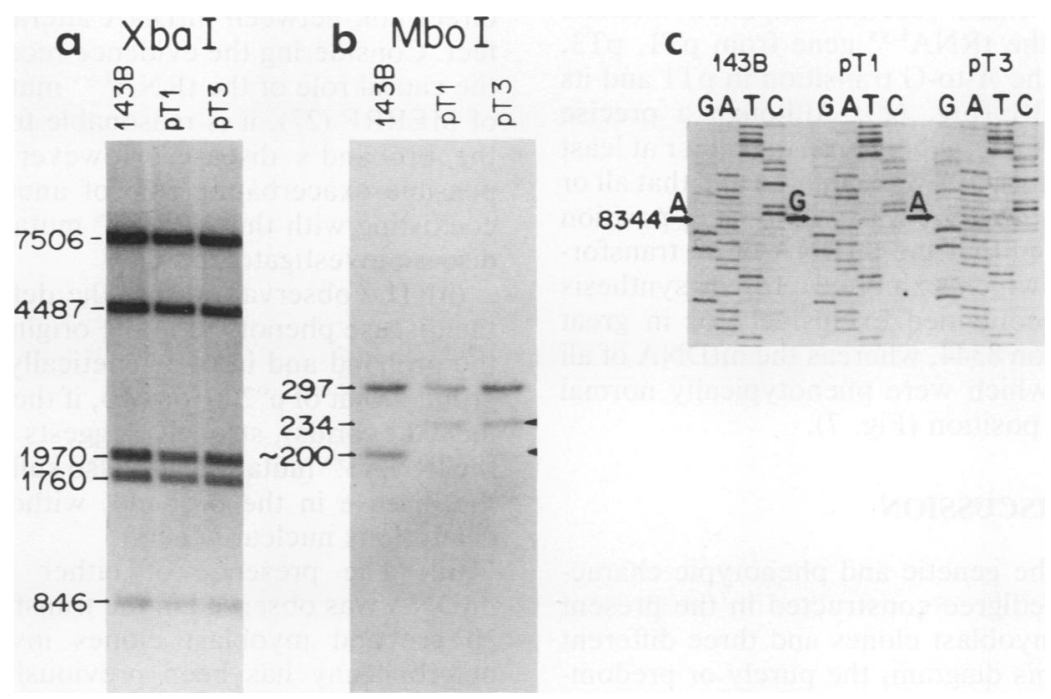

FIG. 6. Analysis of the mtDNA of the transformants. (a) mtDNAs of pT1 and pT3 do not contain any major deletions, as judged from the identity in their $\mathrm{XbaI}$ restriction pattern to that of 143B mtDNA. (b) mtDNAs of pT1 and pT3 share an MboI polymorphism not present in 143B mtDNA. (c) pT1 mtDNA exhibits an A-to-G transition at position 8344 of the tRNA ${ }^{\text {Lys }}$ which is not present in pT3 mtDNA or 143B mtDNA. 


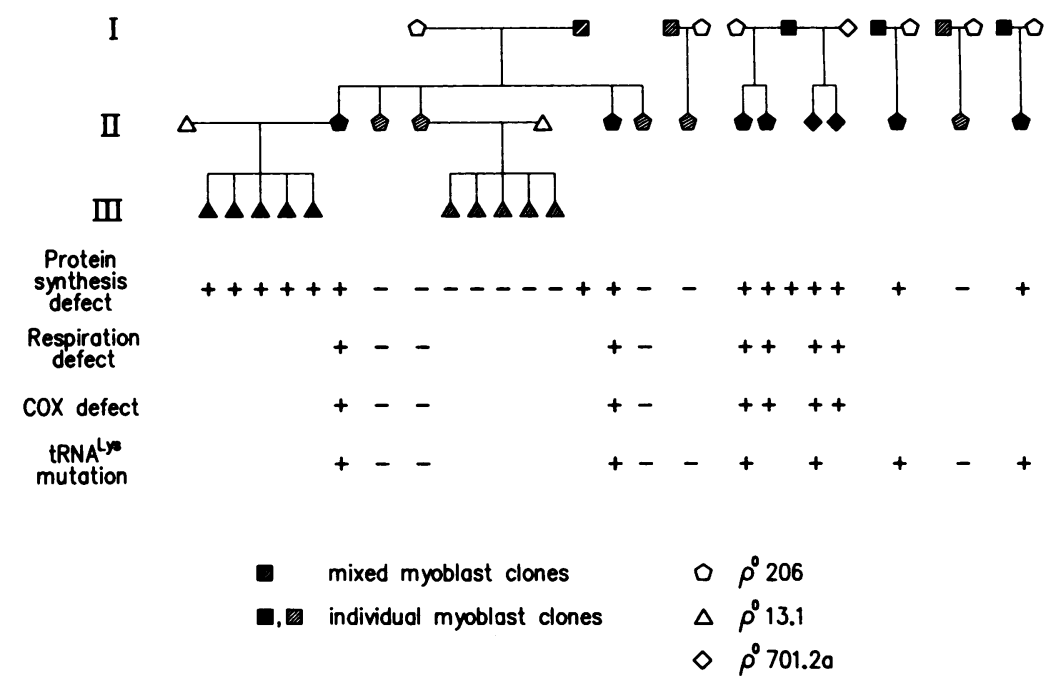

FIG. 7. Phenotypic and genetic characterization of the cellular pedigree constructed in the present work. The solid symbols represent a defective genotype and phenotype; the hatched symbols represent a normal genotype and phenotype. See text for details.

digests (data not shown). In another Southern blot experiment involving $M b o I$, both pT1 and pT3 mtDNA revealed a fragment length polymorphism which distinguished the transformant mtDNA from that of $143 \mathrm{~B} . \mathrm{TK}^{-}$, the parental line of $\rho^{\circ} 206$ (Fig. 6b). It is also clear from the blots in Fig. 6 that the pT1 and pT3 transformants contain approximately equivalent amounts of mtDNA; these amounts are also similar to the amount of mtDNA in 143B cells, previously determined to be $\sim 9,100$ molecules per cell (15).

Very recently, a report (27) has appeared linking the occurrence of an mtDNA tRNA ${ }^{\text {Lys }}$ A-to-G transition at position 8344 (1) to the MERRF syndrome. Since two members of the proband family investigated in the present work exhibited a full MERRF syndrome, and since the proband myoblasts and the transformant cultures derived therefrom showed a protein synthesis defect, it seemed possible that the mtDNA mutation causing this defect was a tRNA $^{\text {Lys }}$ mutation at position 8344 . Indeed, sequence analysis of the polymerase chain reaction-amplified mtDNA fragment encompassing the tRNA ${ }^{\text {Lys }}$ gene from pT1, pT3, and $143 \mathrm{~B}$ cells revealed the A-to-G transition in pT1 and its absence in pT3 and 143B (Fig. 6c). Although a precise quantitation could not be done, it appeared that all or at least the great majority of pT1 mtDNA was mutated and that all or the great majority of pT3 mtDNA was wild type at position 8344. Further tests showed that the mtDNA of all transformants thus investigated which exhibited protein synthesis and respiratory defects contained exclusively or in great predominance $G$ at position 8344 , whereas the mtDNA of all transformants analyzed which were phenotypically normal contained $\mathrm{A}$ at the same position (Fig. 7).

\section{DISCUSSION}

Figure 7 summarizes the genetic and phenotypic characteristics of the cellular pedigree constructed in the present work with the proband myoblast clones and three different $\rho^{\circ}$ human cell lines. In this diagram, the purely or predominantly defective genotype and phenotype of the original individual myoblast clones was inferred from that of the transformants derived therefrom. For this inference, the plausible assumption was made, in agreement with previous evidence $(15,30)$, that there was no intracellular and/or intercellular selective advantage of mutated, nonfunctional mtDNA over wild-type mtDNA during in vitro growth. The correctness of this assumption was directly verified, at least as concerns the phenotype, for one of the clones (no. 2). On the contrary, the assignment of a wild-type genotype and phenotype to some of the original myoblast clones did not exclude the presence in these of a portion of mutated mtDNA which could have been lost by selection during in vitro growth.

Several conclusions can be drawn from the data of Fig. 7. (i) The perfect correlation observed in the transformants between the presence or absence of a protein synthesis defect, an $\mathrm{O}_{2}$ consumption defect, and COX deficiency, on the one hand, and the presence or absence of the mitochondrial tRNA ${ }^{\text {Lys }}$ gene mutation, on the other hand, shows that the mtDNA carrying this mutation was at least in part responsible for the defective phenotype, thus establishing a direct link between mtDNA alteration and biochemical defect. Considering the evidence recently reported concerning the causal role of the tRNA ${ }^{\text {Lys }}$ mutation in the pathogenesis of MERRF (27), it is reasonable to assume a similar role in the proband's disease. However, one cannot exclude a possible exacerbating role of another mtDNA mutation(s) coexisting with the tRNA ${ }^{\text {Lys }}$ mutation in the genesis of the disease investigated here.

(ii) The observation that the defective mtDNA produced the disease phenotype in the original nuclear background of the proband and in one genetically unrelated nuclear background, that of $\rho^{\circ} 206$ (or two, if the 701.2a transformants are indeed cybrids), strongly suggests that the mtDNA carrying the tRNA ${ }^{\text {Lys }}$ mutation was also sufficient by itself to produce the disease in the proband, without the involvement of a deleterious nuclear gene(s).

(iii) The presence of either mutated or nonmutated mtDNA was observed in the transformants derived from the 10 proband myoblast clones investigated here. mtDNA heteroplasmy has been previously reported in tissues or blood in other cases of mitochondrial disease $(11-13,23,27$, $29,34)$. In an analysis of clonal cultures from patients with Kearns-Sayre syndrome, individual muscle or fibroblast clones were found to contain either only normal mtDNA or 
a heteroplasmic population of normal and deleted mtDNA, with occasional clones containing a high proportion (60 to $70 \%$ ) of deleted mtDNA (19). In the present work, heteroplasmy in the myoblasts was probably to a large extent intercellular. The data obtained, in fact, suggest that at least $50 \%$ of the myoblasts in the original myoblast culture contained exclusively or in great predominance defective mtDNA. This figure agrees roughly with the percentages of ragged red fibers and $\mathrm{COX}$-negative fibers determined in the histochemical analysis of the muscle biopsy specimen. The possibility of analyzing both defective and normal myoblasts from the same individual has provided a powerful tool in the present work to correlate the defective genotype with the defective phenotype. Since the selection scheme used for the isolation of transformants depended on the presence of functional mitochondria in the cytoplasts, one must conclude that either the mutated mtDNA molecules allowed a minimal level of respiratory function in the defective myoblasts or that these myoblasts contained a small amount of wild-type mtDNA not detectable in the DNA sequence analysis performed here.

It seems very likely that the $\operatorname{TRNA}^{\mathrm{Lys}}$ mutation caused the disease phenotype in the proband by severely inhibiting mitochondrial protein synthesis in the myoblasts, and probably in other cells, and thus by drastically affecting the assembly of respiratory complexes. In favor of this mechanism of action of the tRNA ${ }^{\text {Lys }}$ mutation is the observation that, although the synthesis of all polypeptides in the defective transformants was severely affected, there was a tendency for this inhibition to be more complete the higher the number of lysine residues in the polypeptides (data not shown). The abnormal translation products observed in the defective myoblasts and transformants derived therefrom most probably represent incomplete polypeptides resulting from premature termination. Consistent with this interpretation is the finding that the abnormal polypeptide migrating faster than ND1 (Fig. 2b and 3) was precipitable by antibodies directed against the $\mathrm{NH}_{2}$-terminal octapeptide of ND2 (data not shown). The observed inhibition of activity of the respiratory chain enzyme $\mathrm{COX}$ is most probably a consequence of the reduced rate of assembly of the COX complex, owing to the drastically curtailed synthesis of the mtDNAencoded subunits I, II, and III, and is therefore consistent with the interpretation discussed above of the mechanism of action of the tRNA ${ }^{\text {Lys }}$ mutation.

The tRNA ${ }^{\text {Lys }}$ mutation affects a conserved nucleotide in the T $\Psi C$ loop (27). This loop is known to reside, with the D loop, at the corner of the L-shaped tridimensional structure of the tRNAs (14) and to be involved in the interaction of the tRNA with the ribosomal surface (24). Therefore, it seems possible that the mutation at position 8344 affects protein synthesis by interfering with the binding of the charged tRNA $^{\text {Lys }}$ to the ribosome. On the other hand, in view of what is known about the specific interaction of the aminoacyl-tRNA synthetases with the tRNA molecules (21), one cannot exclude that the mutation affects the charging of the tRNA ${ }^{\text {Lys }}$. A third possibility is that the mutation interferes with the processing of the tRNA ${ }^{\text {Lys }}$ and thereby affects its stability. In any case, it is clear that the availability of established transmitochondrial cell lines carrying the tRNA $^{\text {Lys }}$ mutation makes the mechanism of this mutation accessible to investigation.

The development of cellular models of mitochondrial diseases, like that described in the present work, will open the way to the analysis of the genetic origin of the defect in other diseases, to the investigation of the role of mtDNA heteroplasmy and of the nuclear background in the penetrance of the disease, to the molecular dissection of the defect, and, in the future, to the establishment of rational therapeutic approaches for the treatment of the disease.

\section{ACKNOWLEDGMENTS}

These investigations were supported by Public Health Service grant GM-11726 to G.A. from the National Institutes of Health and by a grant from the "Associazione Amici Centro Dino Ferrari" to G.M., N.B., and G.S.

We thank Barbara Wold for the 701.2.8c cell line; Michael King for the M13mp18 clone, for carrying out the transformation of one of the control myoblast cultures, and for technical advice; and Benneta Keeley, Arger Drew, and Lisa Tefo for technical assistance.

\section{REFERENCES}

1. Anderson, S., A. T. Bankier, B. G. Barrell, M. H. L. de Bruijn, A. R. Coulson, J. Drouin, I. C. Eperon, D. P. Nierlich, B. A. Roe, F. Sanger, P. H. Schreier, A. J. H. Smith, R. Staden, and I. G. Young. 1981. Sequence and organization of the human mitochondrial genome. Nature (London) 290:457-465.

1a.Attardi, G., B. Keeley, and S. T. Lai. Unpublished data.

2. Attardi, G., M. P. King, A. Chomyn, and P. Loguercio Polosa. 1990. In T. Sato (ed.), Mitochondrial encephalomyopathies, in press. Raven Press, New York.

3. Attardi, G., and G. Schatz. 1988. Biogenesis of mitochondria. Annu. Rev. Cell Biol. 4:289-333.

4. Bresolin, N., L. Bet, M. Moggio, C. Ponzetto, A. Prelle, A. Gallanti, P. Cerretelli, E. Ciafaloni, and G. Scarlato. 1989. Unusual features in a family with mitochondrial myopathy. Neurology 39(Suppl. 1):259.

4a.Bresolin, N., G. Meola, M. Moggio, C. Ponzetto, and G. Scarlato. Unpublished data.

5. Capaldi, R. A. 1988. Mitochondrial myopathies and respiratory chain proteins. Trends Biochem. Sci. 13:144-148.

6. Ching, E., and G. Attardi. 1982. High resolution electrophoretic fractionation and partial characterization of the mitochondrial translation products from HeLa cells. Biochemistry 21:31883195.

7. Chomyn, A., and S. T. Lai. 1990. Regulation of expression of nuclear and mitochondrial genes for mammalian NADH dehydrogenase, p. 179-185. In E. Quagliariello, S. Papa, F. Palmieri, and C. Saccone (ed.), Structure, function and biogenesis of energy transfer systems. Elsevier Biomedical Press, Amsterdam.

8. DiMauro, S., E. Bonilla, M. Zeviani, M. Nakagawa, and D. C. DeVivo. 1985. Mitochondrial myopathies. Ann. Neurol. 17:521538.

9. Feinberg, A. P., and B. Vogelstein. 1983. A technique for radiolabeling DNA restriction endonuclease fragments to high specific activity. Anal. Biochem. 132:6-13.

10. Fung, C. H., and A. K. Khachadurian. 1980. Suppression of synthesis and esterification of cholesterol and stimulation of low density lipoprotein receptor activity by polyoxyethylated cholesterol in cultured human fibroblasts. J. Biol. Chem. 255:676680.

11. Holt, I. J., A. E. Harding, and J. A. Morgan-Hughes. 1988. Deletions of muscle mitochondrial DNA in patients with mitochondrial myopathies. Nature (London) 331:717-719.

12. Holt, I. J., D. H. Miller, and A. E. Harding. 1989. Genetic heterogeneity and mitochondrial DNA heteroplasmy in Leber's hereditary optic neuropathy. J. Med. Genet. 26:739-743.

13. Holt, I. J., A. E. Harding, R. K. H. Petty, and J. A. MorganHughes. 1990. A new mitochondrial disease associated with mitochondrial DNA heteroplasmy. Am. J. Hum. Genet. 46:428433.

14. Kim, S. H., F. L. Suddath, G. J. Quigley, A. McPherson, J. L. Sussman, A. H. J. Wang, N. C. Seeman, and A. Rich. 1974. Three-dimensional tertiary structure of yeast phenylalanine transfer RNA. Science 185:435-440.

14a.King, M., and G. Attardi. Unpublished data.

15. King, M. P., and G. Attardi. 1989. Human cells lacking mtDNA: 
repopulation with exogenous mitochondria by complementation. Science 246:500-503.

16. Meola, G., E. Scarpini, M. Velicogna, A. Mottura, P. L. Baron, S. Beretta, and G. Scarlato. 1986. Analysis of fibronectin expression during human muscle differentiation. Basic Appl. Histochem. 30:153-163.

17. Meola, G., M. Velicogna, C. Brigato, A. Bordoni, N. Bresolin, and G. Scarlato. 1986. Clonal analysis and differentiative properties of adult human muscle cells. Cell Biol. Int. Rep. 3:211212.

18. Mitchell, C. H., and G. Attardi. 1978. Cytoplasmic transfer of chloramphenicol resistance in a human cell line. Somatic Cell Genet. 4:737-744.

19. Moraes, C. T., E. A. Schon, S. DiMauro, and A. F. Miranda. 1989. Heteroplasmy of mitochondrial genomes in clonal cultures from patients with Kearns-Sayre syndrome. Biochem. Biophys. Res. Commun. 160:765-771.

20. Morgan-Hughes, J. A. 1986. Mitochondrial diseases. Trends Neurosci. 9:15-19.

21. Normanly, J., and J. Abelson. 1989. tRNA identity. Annu. Rev. Biochem. 58:1029-1049.

22. Oliver, N. A., B. D. Greenberg, and D. C. Wallace. 1983. Assignment of a polymorphic polypeptide to the human mitochondrial DNA unidentified reading frame 3 gene by a new peptide mapping strategy. J. Biol. Chem. 258:5834-5839.

23. Ozawa, T., M. Yoneda, M. Tanaka, K. Ohno, W. Sato, H. Suzuki, M. Nishikimi, M. Yamamoto, I. Nonaka, and S. Hori. 1988. Maternal inheritance of deleted mitochondrial DNA in a family with mitochondrial myopathy. Biochem. Biophys. Res. Commun. 154:1240-1247.

24. Rich, A., and U. L. RajBhandary. 1976. Transfer RNA: molecular structure, sequence, and properties. Annu. Rev. Biochem. 45:805-860.

25. Rosing, H. S., L. C. Hopkins, D. C. Wallace, C. M. Epstein, and
K. Weidenheim. 1985. Maternally inherited mitochondrial myopathy and myoclonic epilepsy. Ann. Neurol. 17:228-237.

26. Sanger, F., S. Nicklen, and A. R. Coulson. 1977. DNA sequencing with chain-terminating inhibitors. Proc. Natl. Acad. Sci. USA 74:5463-5467.

27. Shoffner, J. M., M. T. Lott, A. M. S. Lezza, P. Seibel, S. Ballinger, and D. C. Wallace. 1990. Myoclonic epilepsy and ragged-red fiber disease (MERRF) is associated with a mitochondrial DNA tRNA ${ }^{\text {Lys }}$ mutation. Cell 61:931-937.

28. Storrie, B., and G. Attardi. 1972. Expression of the mitochondrial genome in HeLa cells. XIII. Effect of selective inhibition of cytoplasmic or mitochondrial protein synthesis on mitochondrial nucleic acid synthesis. J. Mol. Biol. 71:177-199.

29. Vilkki, J., M.-L. Savontaus, and E. K. Nikoskelainen. 1989. Genetic heterogeneity in Leber optic neuroretinopathy revealed by mitochondrial DNA polymorphism. Am. J. Hum. Genet. 45:206-211.

30. Wallace, D. C. 1986. Mitotic segregation of mitochondrial DNA in human cell hybrids and expression of chloramphenicol resistance. Somatic Cell Mol. Genet. 12:41-49.

31. Wallace, D. C. 1989. Mitochondrial DNA mutations and neuromuscular disease. Trends Genet. 5:9-13.

32. Wallace, D. C., G. Singh, M. T. Lott, J. A. Hodge, T. G. Schurr, A. M. S. Lezza, L. J. Elsas II, and E. K. Nikoskelainen. 1988. Mitochondrial DNA mutation associated with Leber's hereditary optic neuropathy. Science 242:1427-1430.

33. Wiseman, A., and G. Attardi. 1982. Cytoplasmically determined human cell mutants defective in mitochondrial ribosome assembly. Mol. Gen. Genet. 186:364-371.

33a.Wold, B. Personal communication.

34. Zeviani, M., C. T. Moraes, S. DiMauro, H. Nakase, E. Bonilla, E. A. Schon, and L. P. Rowland. 1988. Deletions of mitochondrial DNA in Kearns-Sayre syndrome. Neurology 38:13391346. 